\title{
Automated ensemble segmentation of epithelial proliferation, necrosis and fibrosis using scatter tumor imaging
}

\author{
P. Beatriz Garcia-Allende ${ }^{a}$, Olga M. Conde ${ }^{a}$, Venkataramanan Krishnaswamy ${ }^{b}$, P. Jack \\ Hoopes $^{b c}$, Brian W. Pogue ${ }^{b}$, Jesus Mirapeix ${ }^{a}$ and Jose M. Lopez-Higuera ${ }^{a}$ \\ ${ }^{a}$ Photonics Engineering Group, University of Cantabria, Avda. Los Castros s/n, 39005 \\ Santander, Spain; \\ ${ }^{b}$ Thayer School of Engineering, Dartmouth College, Hanover, NH-USA, 03755; \\ ${ }^{c}$ Department of Surgery, Dartmouth Medical School, Hanover, NH-USA, 03755
}

\begin{abstract}
Conventional imaging systems used today in surgical settings rely on contrast enhancement based on color and intensity and they are not sensitive to morphology changes at the microscopic level. Elastic light scattering spectroscopy has been shown to distinguish ultra-structural changes in tissue. Therefore, it could provide this intrinsic contrast being enormously useful in guiding complex surgical interventions.

Scatter parameters associated with epithelial proliferation, necrosis and fibrosis in pancreatic tumors were previously estimated in a quantitative manner. Subtle variations were encountered across the distinct diagnostic categories. This work proposes an automated methodology to correlate these variations with their corresponding tumor morphologies. A new approach based on the aggregation of the predictions of K-nearest neighbors $(\mathrm{kNN})$ algorithm and Artificial Neural Networks (ANNs) has been developed. The major benefit obtained from the combination of the distinct classifiers is a significant increase in the number of pixel localizations whose corresponding tissue type is reliably assured. Pseudo-color diagnosis images are provided showing a strong correlation with sample segmentations performed by a veterinary pathologist.
\end{abstract}

Keywords: automatic classification, tumor, necrosis, confocal reflectance imaging, scatter, feature extraction, k-nearest neighbors (kNN), Artificial neural networks (ANN)

\section{INTRODUCTION}

The quantification of scatter origin features in tissue has been successfully accomplished by angle resolved or coherence based reflectance methods. ${ }^{1,2}$ The measurement can be robust and changes in scatter spectra are related to pathologic structures which occur in the tissue. Therefore, scatter evaluation could provide an additional diagnosis tool for the delineation of tissue morphology at the microscopic level and helping the guidance of surgical resection.

In a previous study, ${ }^{3}$ scattering changes associated with epithelial proliferation, necrosis and fibrosis in tumors were estimated in a quantitative manner using highly localized measurements of reflectance. Automated methodologies based on k-nearest neighbors $(\mathrm{kNN})$ algorithm $^{4}$ and Artificial Neural Networks (ANNs) ${ }^{5}$ to establish a correlation between the subtle changes encountered and the tissue morphology have been already

Further author information: (Send correspondence to P.B.G.)

P.B.G.: E-mail: garciapb@unican.es; Telephone: +34 9422008 77; http://gif.teisa.unican.es/

Biophotonics: Photonic Solutions for Better Health Care II, edited by Jürgen Popp,

Wolfgang Drexler, Valery V. Tuchin, Dennis L. Matthews, Proc. of SPIE Vol. 7715, 77151B

(C) 2010 SPIE · CCC code: $1605-7422 / 10 / \$ 18 \cdot$ doi: $10.1117 / 12.854559$

Proc. of SPIE Vol. 7715 77151B-1 
reported. ${ }^{6,7}$ Both approaches consisted of two distinct steps: statistical pre-processing of the scattering parameters images to create additional data features ${ }^{6}$ and an automated identification procedure using separately either the $\mathrm{kNN}$ or the ANN classifier algorithm. As a result, pseudo-color diagnosis images were provided showing a strong correlation between the segmentation performed by a veterinary pathologist and the automated one within some predefined regions of interest. A veterinary pathologist analyzed the H\&E stained sections of the measured samples ${ }^{3}$ and identified several regions of interest corresponding to the observed tissue types. These tissue types were classified under three major groups: epithelium, fibrosis and necrosis with some constituent subgroups. According to the exhibited nucleus to cytoplasm ratio, epithelial cells were classified in high and low proliferation index tumor cells, while three different fibrosis subgroups (early, intermediate and mature) were also distinguished. In this work, an ensemble method, which consists in the aggregation of the predictions of the $\mathrm{kNN}$ - and the ANN-based segmentations, is proposed. The major benefit obtained from the combination of the distinct classifiers is a significant increase in the number of pixel localizations whose corresponding tissue type is reliably assured.

\section{EXPERIMENTAL ISSUES}

\subsection{Scatter imaging system and parameter fitting}

The scatter scanning system consisted of a confocal spectroscopic system having illumination and detection spot sizes smaller than one scattering length (typically $100 \mu \mathrm{m}$ for tissue ${ }^{8}$ ), and a raster-scanning platform built using linear translation stages. This spot size was specifically chosen as it provides a scatter signal which does not have significant multiple scatter, making it essentially linearly dependent upon the scatter coefficient. A schematic and a detailed description of the system can be found in this previous paper. ${ }^{3}$

The acquired spectral reflectance is fitted by the empirical relationship, which accounts for scatter versus wavelength, and then exponential attenuation to absorption.

$$
I_{R}(\lambda)=A \lambda^{-b} e^{-k c\left\{d\left[H b O_{2}(\lambda)+(1-d) H b(\lambda)\right]\right\}}
$$

where $A$ is the scattered amplitude, $b$ the scattering power, $c$ is proportional to the concentration of whole blood and $d$ is the oxygen saturation fraction. This relationship is valid in the presence of significant local absorption, for very small source-detector separation and when the scattering and absorption coefficients are within the typical range found in tissue. ${ }^{9}$ The extinction spectra of oxygenated and de-oxygenated hemoglobin, $\mathrm{HbO}_{2}(\lambda)$ and $H b(\lambda)$, were obtained from a medical data base. Absorption from other chromophores were assumed to be negligible in the wavelength range of interest, from 510 to $785 \mathrm{~nm}$, and the path length $k$ is assumed to be a wavelength independent constant. Along with the scattered amplitude and the scattering power, a third scattering parameter of interest for tissue discrimination purposes was the average scattered irradiance, $I_{\text {avg }}$, which was obtained by integrating $I_{R}$ over all wavelengths beyond $610 \mathrm{~nm}$ to avoid the strong hemoglobin absorption peaks.

\subsection{Pancreatic tumors}

Data from AsPC-1 pancreatic tumor samples from the previous study ${ }^{3}$ was used. In this regard, human pancreatic tumor cells AsPC-1 were grown and injected subcutaneously in the flank region of male mice. Tumors were harvested seven weeks after injection when they measured $6-7 \mathrm{~mm}$ in diameter and $5-6 \mathrm{~mm}$ in thickness. Then, they were dissected into $4-5 \mathrm{~mm}$ thick sections and imaged. In total, six tumor tissue sections harvested 

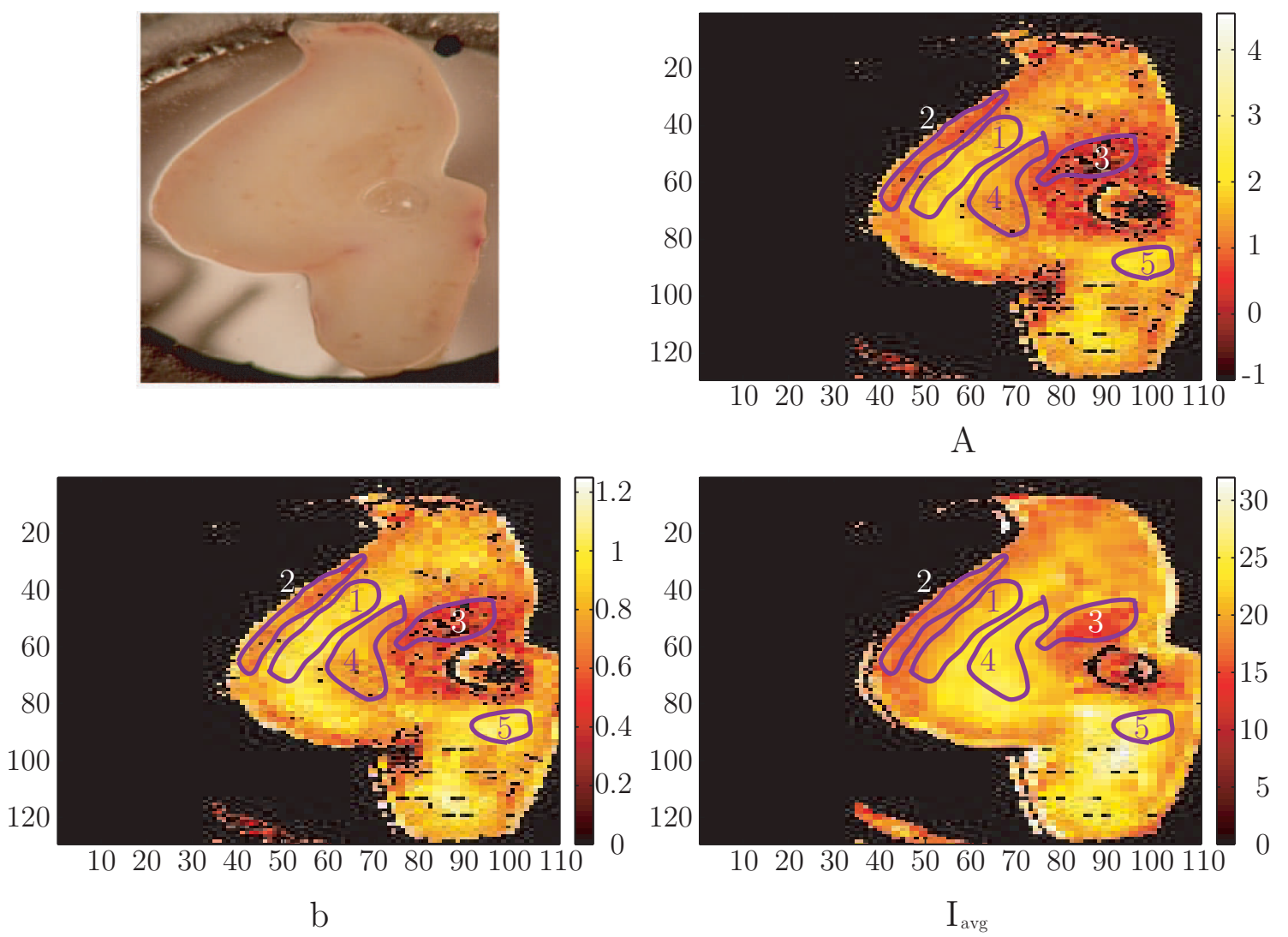

Figure 1. Scattered amplitude $(A)$, scattering power $(b)$ and average scattered irradiance $\left(I_{a v g}\right)$ images of a pancreas tumor sample where five regions-of-interest have been identified.

from four mice were imaged. After the measurement, the sample was routinely processed for subsequent histology evaluation by the veterinary pathologist. Figure 1 depicts one of the analyzed pancreas tumor samples, where five regions-of-interest are shown overlaid on the scattering parameter images (scattered amplitude $A$, scattering power $b$ and average scattered irradiance $I_{a v g}$ whose color scale bars are shown on the right of each corresponding image). Tumor cells found in Region 1 have less cellular density (LPI) than the HPI tumor cells found in Region 2. Region 3 exhibits necrosis and Regions 4 and 5 early and intermediate fibrosis, respectively. Pixels in black correspond to locations where the scatter data could not be reliably measured and they are tagged from now on as masked pixels.

\section{TUMOR REGION SEGMENTATION METHODS}

\subsection{K-nearest neighbors}

For classification purposes, every pixel inside the pre-defined regions of interest is considered as a vector in a 3-dimensional space, the so-called feature space. The three measured scatter parameters $A, b$ and $I_{\text {avg }}$, are each considered a coordinate axis, and the distance between points in this Cartesian space defines how similar or different they are. Since kNN is based on the idea that similar data should belong to the same class, the $k$ pixels with the most similar scattering parameters to an unclassified pixel are initially determined, and this similarity 
is measured in terms of the Euclidean distance, $D$, expressed as:

$$
D\left(p_{1}, p_{2}\right)=\sqrt{\left(A_{1}-A_{2}\right)^{2}+\left(b_{1}-b_{2}\right)^{2}+\left(I_{a v g_{1}}-I_{a v g_{2}}\right)^{2}}
$$

where $p_{1}$ and $p_{2}$ are the two compared tissue pixel localizations. The unclassified pixel is assigned to the most numerous tissue sub-type (high or low proliferation index epithelium, necrosis, and early, intermediate or mature fibrosis) among the closest $k$ neighbors.

\subsection{Additional statistical feature extraction}

The segmentation capacity of the kNN methodology as described above was weak, since six different tissue morphologies had to be discriminated from a data set that lay in a 3-dimensional space. The dimensionality of the data feature space was increased by the addition of a high-order statistical calculation. The first four statistical moments: mean $(\bar{p})$, standard deviation $\left(\sigma_{p}\right)$, skewness $\left(S_{p}\right)$ and kurtosis $\left(K_{p}\right)$, of each scattering parameter $\left(A, b, I_{\text {avg }}\right)$ were calculated over a square spatial vicinity region centered in each pixel localization. Skewness is a measure of the asymmetry of the data around the class mean, while kurtosis is a measure of how outlier-prone a class distribution is. ${ }^{10}$ Then, the statistical moments were concatenated with the three fitted scattering parameters to form a 15-dimensional feature space. Consequently, Equation 2 becomes in this 15-dimensional space:

$$
D\left(p_{1}, p_{2}\right)=\sqrt{\sum_{i=1}^{15}\left(p_{1_{i}}-p_{2_{i}}\right)^{2}}
$$

Figure 2 presents the distribution images of the statistical moments of the scattering parameters, that correspond to the same tumor sample that the one showed in Fig. 1. Figure 2 suggests that the most discriminant parameter is the mean of the scattering power followed by the mean of the averaged intensity. In order to mathematically prove the validity of this assumption, a further study on the capability of both the scattering parameters and their extracted statistics (mean, standard deviation, skewness and kurtosis) to discriminate the different tumor regions was also performed. In this study, the sequential floating forward selection (SFFS) algorithm ${ }^{11}$ was used. SFFS is widely applied to reduce the dimensionality (i.e. the number of features) of spectral data prior to interpretation. ${ }^{12,13}$ When processing spectral data, feature refers to each spectral band and the aim of SFFS in this case is to select the $M$ spectral bands that best discriminate among the subject classes, out of the total number $N$ initial bands, so $M<N$. The discrimination among the classes, or class separability, can be calculated performing different statistical computations. ${ }^{12}$ The same fundamental is employed to sort the scattering parameters and their statistical values according to their subtype discrimination capability. In this way, the first feature selected by the algorithm will be the one with the greatest changes according to the pathology. These changes have been measured in terms of the Bhattacharya statistical distance. ${ }^{12}$ Therefore, the difference in a scattering parameter $p$, with $p=1,2, \ldots, 15$, between two tissue subtypes, $i$ and $j$, is given by:

$$
J_{i j}^{p}=\frac{1}{4}\left(\mu_{j}-\mu_{i}\right)^{T}\left[\Sigma_{i}+\Sigma_{j}\right]^{-1}\left(\mu_{j}-\mu_{i}\right)+\frac{1}{2} \ln \frac{\left|\Sigma_{i}+\Sigma_{j}\right|}{2\left(\left|\Sigma_{i}\right|\left|\Sigma_{j}\right|\right)^{1 / 2}}
$$

where $\mu_{i}$ and $\Sigma_{i}$ are the mean and the variance matrix of $p$ for tissue subtype $i$. Because there are six different tissue subtypes, the global class separability measurement, $J$, requires one to calculate the difference between every two subtypes

$$
J=\sum_{i=1}^{6} \sum_{j=1}^{6} P_{i} P_{j} J_{i j}
$$




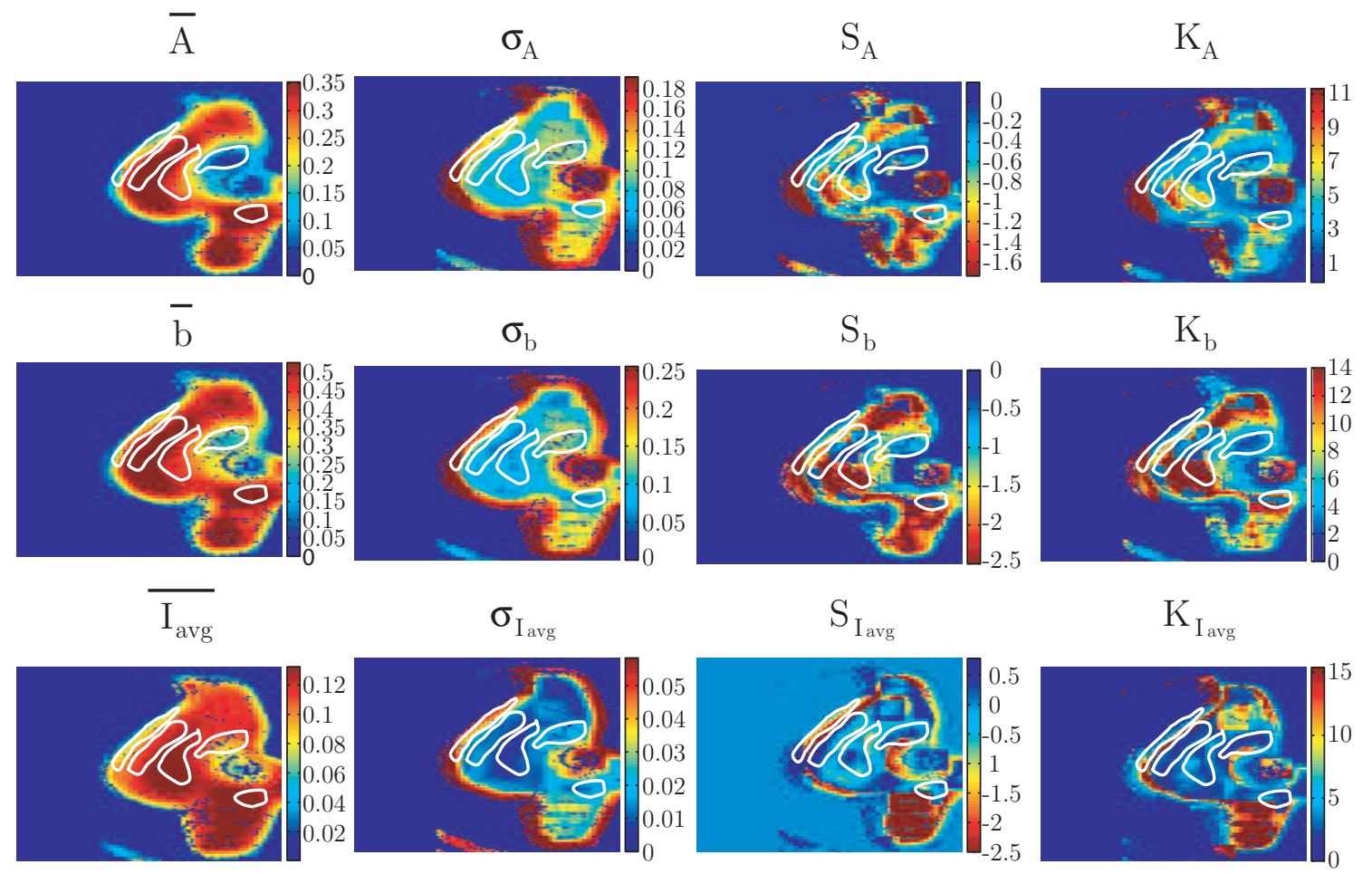

Figure 2. Images of the first four statistical moments: mean $(\bar{p})$, standard deviation $\left(\sigma_{p}\right)$, skewness $\left(S_{p}\right)$ and kurtosis $\left(K_{p}\right)$ of each scattering parameter $p$.

where $P_{i}$ is each subtype probability and $J_{i j}$ is the distance between subtypes $i$ and $j$, as stated in Eq. 4 .

Table 1 summarizes tissue subtype separability measurements through the SFFS algorithm as a function of the size of the vicinity region. For small window sizes, which means that included pixel localizations are mostly within the same tissue subtype, the mean scattering power is always selected as the most discriminant feature. This confirms the feeling derived from Fig. 2 and agrees with the remarkable improvement in methodology segmentation capabilities achieved by the addition of statistical data. ${ }^{6}$

\subsection{Artificial neural networks (ANNs)}

Although ANNs offer a flexible way to model nonlinear functions, ${ }^{14}$ insufficient performance was achieved for tumor discrimination based on an ANN with $\left(A, b, I_{\text {avg }}\right)$ data. Due to the improvement obtained with the $\mathrm{kNN}$ classifier once the high-order statistical parameters were included, the application of an ANN classifier with this new data set was also conducted. The validity of a Backpropagation ANN for the segmentation of the distinct tumor regions was shown. ${ }^{7}$ It consists of a 4-layered structure where two hidden layers (50 nodes each) were required between the input and the output layer (6 nodes as the number of tumor subtypes) to achieve similar correlations between the automated and pathologist-based classifications than the ones obtained by kNN.

\subsection{Assembly of the predictions of the kNN and ANN classifiers}

If classifiers lack stability, data modeling could be improved by the employment of ensemble methods. ${ }^{14}$ These are based on the idea of aggregation, a process that consists in the combination of the predictions of a number of different classifiers into a new prediction. The stability of the $\mathrm{kNN}$ and ANN methodologies previously developed 
Table 1. Sorting of the scattering parameters and their statistics as a function of their tissue subtype discrimination capability.

\begin{tabular}{|c|c|c|c|c|c|}
\hline Window size & $\mathbf{1}$ & $\mathbf{2}$ & $\mathbf{3}$ & $\mathbf{4}$ & $\mathbf{5}$ \\
\hline 30 & $\sigma_{A}$ & $\bar{A}$ & $K_{I_{\text {avg }}}$ & $\bar{b}$ & $\overline{I_{\text {avg }}}$ \\
\hline 28 & $\sigma_{A}$ & $\bar{A}$ & $K_{I_{\text {avg }}}$ & $\overline{I_{\text {avg }}}$ & $\bar{b}$ \\
\hline 26 & $\sigma_{A}$ & $\bar{A}$ & $K_{I_{\text {avg }}}$ & $\overline{I_{\text {avg }}}$ & $\bar{b}$ \\
\hline 24 & $\sigma_{A}$ & $\bar{A}$ & $K_{I_{\text {avg }}}$ & $\overline{I_{\text {avg }}}$ & $\bar{b}$ \\
\hline 22 & $K_{b}$ & $K_{I_{\text {avg }}}$ & $\overline{I_{\text {avg }}}$ & $\sigma_{A}$ & $\bar{A}$ \\
\hline 20 & $K_{b}$ & $K_{I_{\text {avg }}}$ & $\overline{I_{\text {avg }}}$ & $\bar{b}$ & $\bar{A}$ \\
\hline 18 & $\bar{b}$ & $\overline{I_{\text {avg }}}$ & $\bar{A}$ & $K_{b}$ & $\sigma_{A}$ \\
\hline 16 & $\bar{b}$ & $\overline{I_{\text {avg }}}$ & $\sigma_{I_{\text {avg }}}$ & $\bar{A}$ & $K_{I_{\text {avg }}}$ \\
\hline 14 & $\bar{b}$ & $\overline{I_{\text {avg }}}$ & $\sigma_{I_{\text {avg }}}$ & $\bar{A}$ & $K_{I_{\text {avg }}}$ \\
\hline 12 & $\bar{b}$ & $\overline{I_{\text {avg }}}$ & $\bar{A}$ & $I_{\text {avg }}$ & $\sigma_{A}$ \\
\hline 10 & $\bar{b}$ & $\overline{I_{\text {avg }}}$ & $\bar{A}$ & $I_{\text {avg }}$ & $\sigma_{A}$ \\
\hline 8 & $\bar{b}$ & $\bar{A}$ & $\overline{I_{\text {avg }}}$ & $\sigma_{A}$ & $I_{\text {avg }}$ \\
\hline 6 & $\bar{b}$ & $\bar{A}$ & $\overline{I_{a v g}}$ & $\sigma_{A}$ & $\sigma_{I_{\text {avg }}}$ \\
\hline 4 & $\bar{b}$ & $\bar{A}$ & $\overline{I_{a v g}}$ & $\sigma_{A}$ & $I_{\text {avg }}$ \\
\hline 2 & $\bar{b}$ & $\bar{A}$ & $\overline{I_{a v g}}$ & $\sigma_{A}$ & $I_{\text {avg }}$ \\
\hline
\end{tabular}

was tested and high accuracies were achieved with independence of the training and test data sets. ${ }^{6,7}$ However, this stability can only be measured within the regions-of-interest defined by the pathologist. It is reasonable to think that discrimination performance outside them will be good too, but it is not possible to conclude it. The latter is examined here by means of a combined method of $\mathrm{kNN}$ and ANN predictions. The procedure is graphically described in Figure 3. Only tissue sub-type assignation is assured in these pixel localizations where tumor sub-type assigned by both methodologies match. Pixels where each methodology concludes a different tumor sub-type are assigned as masked pixels.

\section{RESULTS AND DISCUSSION}

Figure 4 presents the pseudo-color diagnosis images of the tumor sample depicted in Fig. 1 obtained by means of the kNN-based (Fig. 4.a) and the ANN-based (Fig. 4.b) methodologies and the diagnosis image after the application of the combination procedure (Fig. 4.c) described above. The latter is compared with the segmentation of the sample performed by the veterinary pathologist (Fig. 4.d). The segmentation of other analyzed tumor samples in their distinct tumor regions (by means of the ensemble method) is presented in Figure 5 . As expected, an accurate correlation is achieved within the regions-of-interest of all samples, as concluded in previous works. ${ }^{6,7}$ However, the number of pixel localizations reliably assured (by means of two different and independent methodologies) is much higher. Table 2 compares the prediction rates of the ensemble method with the number of pixels per sample classified by the veterinary pathologist, which coincides with the number of pixels reliably assured by the independent kNN and ANN segmentations. The global identification rate is more than five times greater when both methodologies are combined with the additional fact of assurance in the identification provided by this peer methodology. 


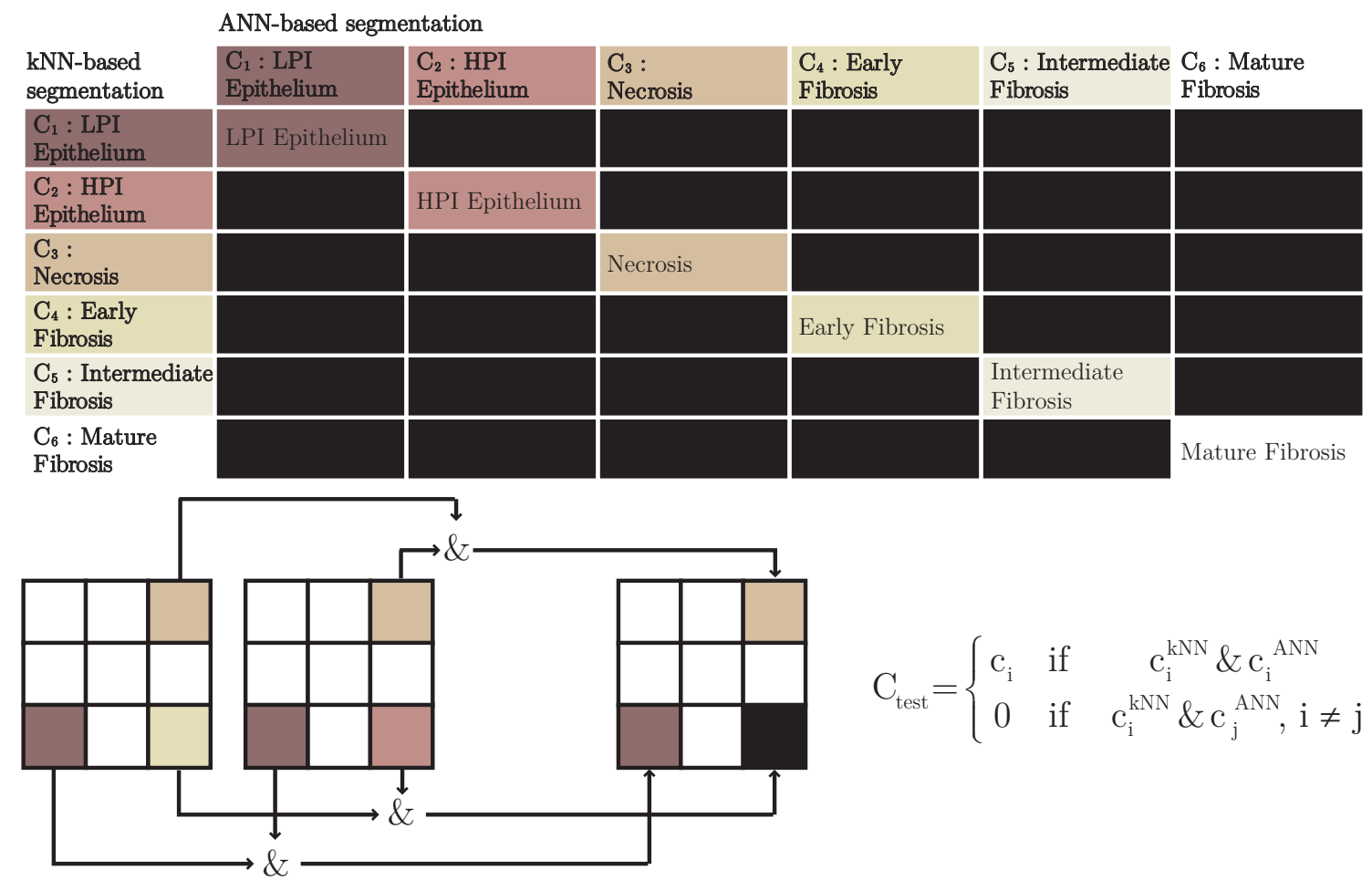

Figure 3. Schematic of the combination of the kNN and ANN predicted tumor subtypes.

Table 2. Sorting of the scattering parameters and their statistics as a function of their tissue subtype discrimination capability.

\begin{tabular}{|l|l|l|l|}
\hline $\begin{array}{l}\text { Tumor sample } \\
\text { no. }\end{array}$ & $\begin{array}{l}\text { Total no. } \\
\text { of pixels }\end{array}$ & $\begin{array}{l}\text { Expert-based } \\
\text { classified pixels }\end{array}$ & $\begin{array}{l}\text { No. of pixels classified } \\
\text { by the aggregated method }\end{array}$ \\
\hline 1 & 5476 & $9.8430 \%(539)$ & $55.1315 \%(3019)$ \\
\hline 2 & 5893 & $17.5632 \%(1035)$ & $64.6190 \%(3808)$ \\
\hline 3 & 8450 & $15.1124 \%(1277)$ & $64.0237 \%(5410)$ \\
\hline 4 & 6900 & $2.5072 \%(173)$ & $45.1449 \%(3115)$ \\
\hline 5 & 5966 & $10.6772 \%(637)$ & $52.9165 \%(3157)$ \\
\hline Mean values & & $11.1406 \%$ & $56.3671 \%$ \\
\hline
\end{tabular}

\section{CONCLUSIONS}

An aggregated automated methodology into what scatter changes in tissue means relative to pathology was developed. It consists of the combination of two different approaches based on $\mathrm{kNN}$ and ANN classifiers, respectively, whose validities for tumor subtype discrimination have been previously demonstrated. However, calculations of the statistical moments of the scattering parameters in a vicinity region of every pixel location were required to achieve a reliable discrimination of the distinct tumor regions, both in kNN- and ANN-based methodologies. In order to justify this requirement, the SFFS algorithm was used to determine which parameters exhibited more consistent trends across the different tumor subtypes. For physically reasonable window sizes, mean scattering parameters are more significant than the parameters themselves, which explains the increase in tumor region determination accuracies. 


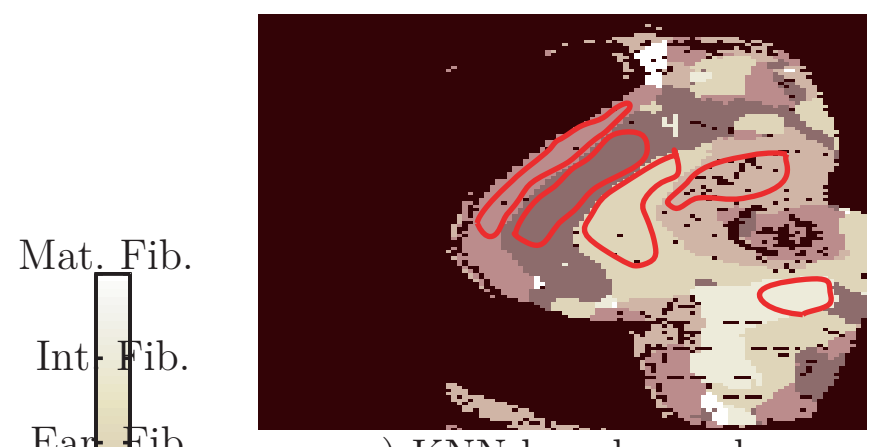

a) KNN-based sample segmentation

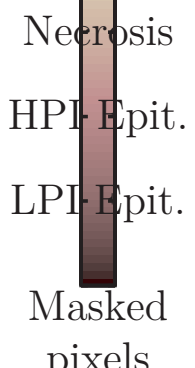

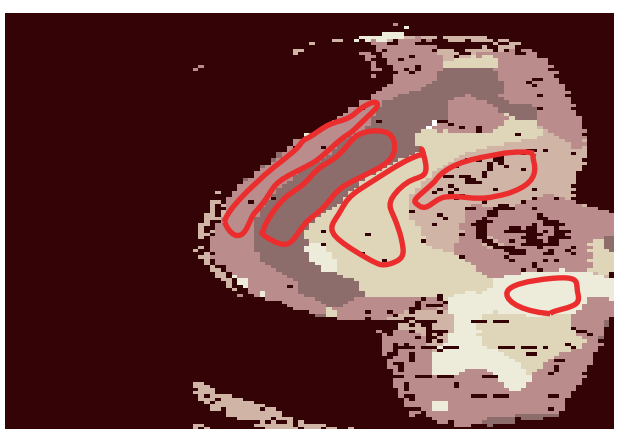

b) ANN-based sample segmentation

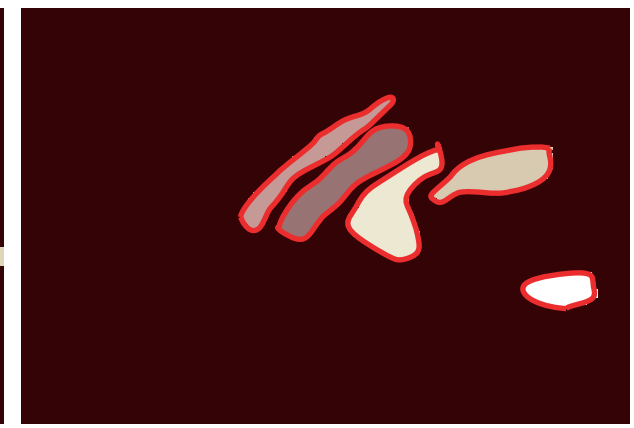

d) Pathologist-based sample segmentation

Figure 4. Qualitative comparison among segmentation of a tumor sample between independent and aggregated methodology (from a) to c)) with the one performed by the veterinary pathologist.

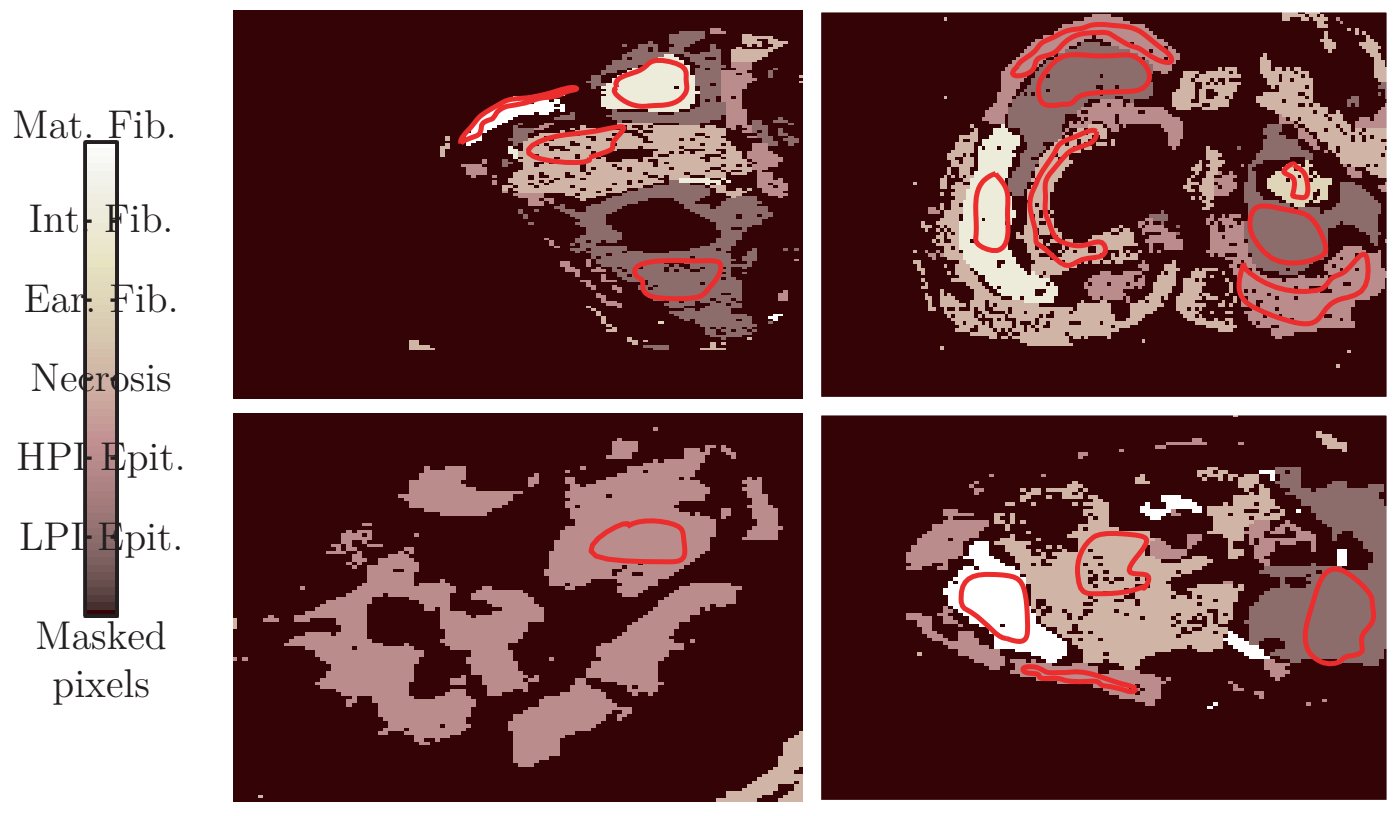

Figure 5. Segmentation performance of some tumor samples is illustrated, employing a 12-pixel window size in statistical calculation, 15-dimensional feature space and a combined kNN-ANN method. 
The major benefit of this peer methodology is that every tissue pixel localization is probed through two distinct and independent methodologies, which enhances the reliability of the concluded tumor subtype. The number of identified pixels is more than five times higher in the segmentations performed by the aggregated method than in the one performed by the pathologist, which implies a significant improvement in the specificity of the tool in tumor region delineation.

Unsupervised classification algorithms are traditionally employed in remote sensing to deal with the typically few and costly labeled data. On account of this, they could also be employed to help with the problem that arises from the fact that the estimation of the ability of the independent methodologies to correlate the scatter changes with their corresponding tissue type is restricted to the number of tissue pixel localizations within the expert predefined regions of interest, although the number of probed pixels in automated manner was much higher. Consequently, future research lines should focus on this approach. Apart from that, the delineation capability of the methodology has only been assessed up to now for pancreatic tumors. Accordingly, ongoing studies are also investigating the viability for prostate and breast tumors.

\section{ACKNOWLEDGMENTS}

P. Beatriz Garcia Allende is grateful for financial support from the Spanish Government's Ministry of Science and Technology through the project TEC'2007-67987-C02-01 allowing her to spend three months in the Thayer

School of Engineering at Dartmouth College. Funding at Dartmouth for this work came from NIH research grants PO1CA84203 and PO1CA80139.

\section{REFERENCES}

1. A. Wax, C. H. Yang, M. G. Muller, R. Nines, C. W. Boone, V. E. Steeleand, G. D. Stoner, R. R. Dasari, and M. S. Feld, "In situ detection of neoplastic transformation and chemopreventive effects in rat esophagus epithelium using angle-resolved low-coherence interferometry," Cancer Res. 63 (13), pp. 3556-9, 2003.

2. A. Wax, "Low-coherence light-scattering calculations for polydisperse size distributions," J. Opt. Soc. Am. A 22 (2), pp. 256-61, 2005.

3. V. Krishnaswamy, P. J. Hoopes, K. S. Samkoe, J. A. O'Hara, T. Hasan, and B. W. Pogue, "Quantitative imaging of scattering changes associated with epithelial proliferation, necrosis, and fibrosis in tumors using microsampling reflectance spectroscopy," J. Biomed. Opt. 14 (1), p. 014004, 2009.

4. K. Fukunaga, Introduction to statistical pattern recogniton, Academic Press, 1990.

5. C. Chen, Fuzzy logic and neural network handbook, McGraw Hill, 1996.

6. P. B. Garcia-Allende, V. Krishnaswamy, P. J. Hoopes, K. S. Samkoe, O. M. Conde, and B. W. Pogue, "Automated identification of tumor microscopic morphology based on macroscopically measured scatter signatures," J. Biomed. Opt. 14 (3), p. 034034, 2009.

7. P. B. Garcia-Allende, V. Krishnaswamy, K. S. Samkoe, P. J. Hoopes, B. W. Pogue, O. M. Conde, and J. M. Lopez-Higuera, "Automated interpretation of scatter signatures aimed at tissue morphology identification," Proc. of SPIE 7368, p. 73681C, 2009.

8. B. W. Pogue and G. C. Burke, "Fiber optic bundle design for quantitative fluorescence measurement from tissue," App. Opt. 37 (31), pp. 7429-36, 1998.

Proc. of SPIE Vol. 7715 77151B-9 
9. A. Amelink and H. J. Sterenborg, "Measurement of the local optical properties of turbid media by differential path-length spectroscpy," App. Opt. 43 (15), pp. 3048-54, 2004.

10. O. Holub and S. Ferreira, "Quantitative histogram analysis of images," Comput. Phys. Commun. 175, pp. 620-3, 2006.

11. J. Kittler, Handbook of Pattern Recognition and Image Processing, Academic Press, 1986.

12. L. Gomez-Chova, L. Calpe, G. Camps-Valls, J. D. Martin, E. Soria, J. Vila, L. Alonso-Corda, and J. Moreno, "Feature selection of hyperspectral data through local correlation and SFFS for crop classification," IEEE Int. Geosci. Remote Sens. Symp. Proc. 1, pp. 555-7, 2003.

13. B. Deronde, P. Kempeneers, and R. M. Forster, "Imaging spectroscopy as a tool to study sediment characteristics on a tidal sandbank in the Westerschelde," Estuar. Coast. Shelf S. 69 (3-4), pp. 580-90, 2006.

14. C. Krafft, G. Steiner, C. Beleites, and R. Salzer, "Disease recognition by infrared and Raman spectroscopy," J. Biophotonics 2 (1-2), pp. 13-28, 2009. 